\title{
论 文磁相变材料专题
}

\section{$\mathrm{Mn}_{50} \mathrm{Ni}_{32-x} \mathrm{Cu}_{x} \mathrm{Co}_{8} \mathrm{Ti}_{10}$ 合金条带马氏体相变和磁热 效应}

张玉希 ${ }^{1}$, 刘小川 ${ }^{2}$, 余广 ${ }^{1,2}$, 郑先明 ${ }^{1,2}$, 万铎建 ${ }^{2}$, 罗小华 ${ }^{1}$, 陈长材 ${ }^{1}$, 马胜灿 ${ }^{1 *}$

1. 江西理工大学稀土学院, 江西省稀土磁性材料与器件重点实验室/江西理工大学稀土磁性材料与器件研究所, 赣州 341000;

2. 江西理工大学材料冶金化学学部, 材料科学与工程学院, 赣州 341000

*联系人, E-mail: mashengcan@jxust.edu.cn, shengcanma801@gmail.com

收稿日期: 2020-07-30; 接受日期: 2020-09-18; 网络出版日期: 2021-04-19

国家自然科学基金(编号：52061014)、江西省自然科学基金重点项目(编号：20192ACB20004)和南京大学固体微结构物理国家重点实验室开 放课题(编号: M32027)资助

摘要本文主要研究了 $\mathrm{Cu}$ 取代和退火等因素对全过渡族 $\mathrm{Mn}_{50} \mathrm{Ni}_{32-x} \mathrm{Cu}_{x} \mathrm{Co}_{8} \mathrm{Ti}_{10}(x=1,2)$ 合金条带马氏体相变行为 和磁热性能的影响. 研究发现, $\mathrm{Cu}$ 取代后, 合金马氏体相变温度往低温方向移动, 且对 $\mathrm{Cu}$ 含量非常敏感; 同时奥氏 体铁磁性增强, 导致相变前后磁化强度突变增大; 发生明显的磁场驱动变磁性马氏体相变; 磁熵变随着 $\mathrm{Cu}$ 含量的 增加而逐渐增大，尤其是有效制冷能力相对于 $\mathrm{Cu}$ 取代前样品成倍增大. 本文以 $x=1$ 样品研究了退火对马氏体相变 的影响, 退火后, 合金马氏体相变变得缓慢, 奥氏体铁磁性减弱, 相变前后磁化强度突变减小, 磁场驱动变磁性变 弱, 由此导致磁熵变大幅度减小, 但由于制冷温区成倍增大, 致使有效制冷能力几乎不减小. 本文从过渡元素取代 导致 $3 d$ 电子之间相互作用的改变和退火导致 $\gamma$ 相的析出分别讨论了 $\mathrm{Cu}$ 取代和退火影响合金马氏体相变的物理 机制.

关键词 马氏体相变, 变磁性, 磁热效应, $\gamma$ 相, $\mathrm{Mn}_{50} \mathrm{Ni}_{32-x} \mathrm{Cu}_{x} \mathrm{Co}_{8} \mathrm{Ti}_{10}$ 合金

PACS: $75.30 . \mathrm{Sg}, 75.20 . \mathrm{En}, 81.30 . \mathrm{Kf}, 75.60 . \mathrm{Nt}, 75.30 . \mathrm{Kz}$

\section{1 引言}

为了有效应对当今世界两大危机：环境恶化和能 源枯竭，基于磁热效应的磁致冷，以其绿色环保、节 能高效等优点，被誉为最有可能替代当今气体压缩制 冷的一种新型固态制冷技术. 磁热效应是指磁性材料 施加和去除外磁场，会发生磁有序度的改变，从而引
起系统摘发生变化, 伴随吸放热的过程 ${ }^{[-4]}$. 磁制冷已 经在低温区实现应用，但室温区磁制冷在固态制冷技 术发展中更具应用潜力和市场价值. 室温区磁制冷的 关键是开发具有大磁热效应的新型制冷工质。自从 Gd-Si-Ge ${ }^{[5]}, \mathrm{La}-\mathrm{Fe}-\mathrm{Si}^{[6]}, \mathrm{Mn}-\mathrm{Fe}-\mathrm{P}-\mathrm{As} \mathrm{s}^{[7]}$ 等著名磁制冷材 料被发现以来，相变材料尤其是一级磁相变材料成为 新型磁制冷材料研发的热点.

引用格式: 张玉希, 刘小川, 余广, 等. $\mathrm{Mn}_{50} \mathrm{Ni}_{32-x} \mathrm{Cu}_{x} \mathrm{Co}_{8} \mathrm{Ti}_{10}$ 合金条带马氏体相变和磁热效应. 中国科学: 物理学 力学 天文学, 2021, 51: 067511 Zhang Y X, Liu X C, Yu G, et al. Martensitic transformation and magnetocaloric effect in $\mathrm{Mn}_{50} \mathrm{Ni}_{32-x} \mathrm{Cu}_{x} \mathrm{Co}_{8} \mathrm{Ti}_{10}$ Heusler alloy ribbons (in Chinese). Sci Sin-Phys Mech Astron, 2021, 51: 067511, doi: 10.1360/SSPMA-2020-0290 
在众多一级磁相变材料中, Ni-Mn基Heusler合金 也被广泛关注和研究 ${ }^{[2-4,8]}$, 尤其在 2004年, Sutou等 人 $^{[9]}$ 发现 In, $\mathrm{Sn}, \mathrm{Sb}$ 可以取代 Ga后, 新型 $\mathrm{Ni}-\mathrm{Mn}-\mathrm{X}$ $(\mathrm{X}=\mathrm{In}, \mathrm{Sn}, \mathrm{Sb}, \mathrm{Al})$ 铁磁形状记忆合金(FSMAs)马氏体 相变及其附近的磁热效应便被广泛研究 ${ }^{[2-4,8,9]}$. 合适成 分的该系列合金马氏体相变既属于热弹性相变, 又属 于铁磁性相变, 因此既可以被温度或应力诱导, 也可 以被磁场驱动. 在温度、磁场等外界因素的激励下, 合金可以发生铁磁奥氏体和弱磁马氏体之间的马氏体 相变(或逆马氏体相变), 晶体结构转变和磁性相变强 烈耦合, 因此, 相变附近表现出很大的磁热效应 ${ }^{[2-4]}$, 同 时也具有很大的机械热效应(弹热、压热)、巨磁 电阻、磁致应变、交换偏置、动力学日禁等物理效 应 $^{[2-4,10-23]}$, 从而在固态制冷、磁激励、人工智能等前 沿和新兴领域都极具应用前景.

人们在对Ni-Mn基FSMAs研究的过程中, 通过多 种途径调控实现铁磁/弱磁性马氏体相变, 比如过渡/ 主族元素取代 ${ }^{[2-4,8-10,14,15,18-23]}$ 、热处理 ${ }^{[11]}$ 、改变快淬 速度 ${ }^{[13]}$ 、间隙位原子掺杂 ${ }^{[16,17]}$ 等. 人们也提出各种机 制解释马氏体相变调控的物理机理, 比如应力弛 豫 $^{[11]}$ 、反位缺陷 ${ }^{[11]} 、 \mathrm{Mn}-\mathrm{Mn}$ 间距 ${ }^{[2]}$ 、价电子浓度 ${ }^{[8]}$ 、 第二相析出 ${ }^{[11,13]}$ 等. 比如, 2009 年, Liu等人 ${ }^{[11]}$ 发现 $2 \mathrm{~h}$ $900^{\circ} \mathrm{C}$ 的退火处理使 $\mathrm{Ni}_{46} \mathrm{Mn}_{35} \mathrm{In}_{14} \mathrm{Co}_{5}$ 合金的马氏体相 变变缓且相变温度异常降低, 磁熵变 $\left(\Delta S_{\mathrm{M}}\right)$ 也随之减 小, 他们把这些变化归因于退火导致 $\gamma$ 相的析出. 2015 年, Ma等人 ${ }^{[13]}$ 发现当快淬速度增大到 $30 \mathrm{~m} / \mathrm{s}$ 时, Ni-Co$\mathrm{Mn}-\mathrm{Sn}$ 合金条带中也析出 $\gamma$ 相, 由此导致合金马氏体相 变温度的急剧降低.

如上所述，传统的Ni-Mn基Heulser合金是由过渡 族和主族元素组成的, 但是在 2015 和 2016 年, Wei等 人 $^{[14,15]}$ 独辟蹊径, 基于 $d-d$ 弱共价杂化的成键特征, 率 先提出并实现了全部由 $3 d$ 过渡金属元素组成的全过渡 族(All- $d$-Metal) 富 $\mathrm{Ni}$ 基 $\mathrm{Ni}_{50-x} \mathrm{Co}_{x} \mathrm{Mn}_{35} \mathrm{Ti}_{15}$ 和富 $\mathrm{Mn}$ 基 $\mathrm{Mn}_{50} \mathrm{Ni}_{40-x} \mathrm{Co}_{x} \mathrm{Ti}_{10}$ Heusler合金新体系. 通过过渡金属 元素Co的替代, 这种新型Ni-Mn基Heulser合金也实现 了铁磁马氏体相变, 且相变附近也表现出同样甚至更

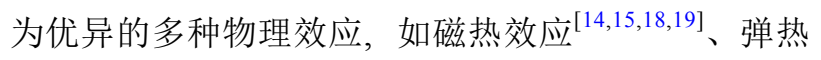
效应 ${ }^{[17,21]}$ 、压热效应 ${ }^{[16,20]}$ 、磁电阻 ${ }^{[14,15,19]}$ 、磁致应 变 ${ }^{[14,15]}$ 、交换偏置 ${ }^{[22]}$ 等, 从而引起人们的广泛重视和 研究. 然而目前对此类合金研究得还不够充分, 且相 关研究大多都集中在富 $\mathrm{Ni}$ 基all- $d$ Ni-Co-Mn-Ti体系的
块材 ${ }^{[14-17,20,21]}$ 、薄膜 ${ }^{[22]}$ 、条带 ${ }^{[18,19,23]}$ 等，人们也通过 设计成分、优化热处理方案等手段对合金的马氏体相 变进行了调控. 然而本文注意到对富 Mn基all- $d \mathrm{Mn}-\mathrm{Ni}$ Co-Ti合金体系, 相关研究仍然很少 ${ }^{[15,23]}$.

相对于富 $\mathrm{Ni}$ 基合金体系，富 $\mathrm{Mn}$ 基all- $d$ 合金采用更 少的 $\mathrm{Co}$ 替代Ni就可以使合金实现剧烈的反铁磁/铁磁 马氏体相变, 从而获得更靠近室温的大 3 倍多的磁致应 变效应 ${ }^{[14,15]}$, 考虑到 $\mathrm{Co}$ 是战略性元素, $\mathrm{Mn}$ 相对便宜, 因 此富 $\mathrm{Mn}$ 基合金体系的成本也相对更低，因此对富 $\mathrm{Mn}$ 基all- $d$ 合金体系马氏体相变及其磁功能性质进行研究 更有利于实际应用 ${ }^{[23]}$. 同时, 相对于块体, 合金条带制 备不需要长时间高温退火即可成相, 成本可以控制得 更低, 更具实际应用意义, 且随着智能社会的发展, 仪 器小型化是大势所趋, 对条带样品进行研究也更具科 学意义, 因此不仅块体, Ni-Mn基合金条带也被人们广 泛重视和深入研究 ${ }^{[11,13,23,24]}$. 基于上述考虑, 本文设计 制备了 $\mathrm{Cu}$ 掺杂的富 $\mathrm{Mn}$ 基all- $d \mathrm{Mn}_{50} \mathrm{Ni}_{32-x} \mathrm{Cu}_{x} \mathrm{Co}_{8} \mathrm{Ti}_{10}$ $(x=1,2)$ 合金条带样品, 通过不同 $\mathrm{Cu}$ 取代量、退火等因 素研究了该系列合金的马氏体相变调控及磁热效应.

\section{2 材料和方法}

名义成分为 $\mathrm{Mn}_{50} \mathrm{Ni}_{32-x} \mathrm{Cu}_{x} \mathrm{Co}_{8} \mathrm{Ti}_{10}(x=1,2)$ 的合金 条带(分别命名为 $\mathrm{Cu} 1, \mathrm{Cu} 2$ ) 通过电弧熔炼与熔体快淬 技术制备得到，所使用的金属单质纯度均在 $99.9 \%$ 以 上. 首先将配制好的原料放在水冷式铜坩埚电弧熔炼 炉中, 在 $\mathrm{Ar}$ 气氛保护下, 熔炼成纽扣状铸锭. 为了保证 合金成分均匀, 需要反复熔炼4-5遍. 考虑到 $\mathrm{Mn}$ 元素易 挥发, 配料过程中多添加 $3 \mathrm{wt}$ \% \% 的 Mn, 以保证熔炼完 成后合金的化学成分基本不偏析. 然后将熔炼好的样 品切成合适的大小, 并打磨掉表面的氧化层, 放在石 英管内, 利用高真空熔体快淬设备在 $\mathrm{Ar}$ 气氛保护下, 以 $15 \mathrm{~m} / \mathrm{s}$ 的速度制备成相应的条带样品. 随后选取适 量的条带样品封入通入少量 $\mathrm{Ar}$ 气的石英管内, 在 $1103 \mathrm{~K}$ 温度下保温 $10 \mathrm{~min}$ 后在冷水中快淬. 利用 $\mathrm{X}$ 射 线衍射(XRD, BRUKER/AXS, 德国)分析样品的晶体 结构, 利用场发射扫描电子显微镜(FESEM, QUANTA200, FEI, 美国)对样品的微观形貌进行表征, 利用 综合物性测试系统(PPMS Quantum Design, 美国)测量 样品的热磁 $M(T)$ 曲线和等温磁化 $M(H)$ 曲线. 其中 $M(T)$ 曲线采用场冷(Field Cooling, FC)/零场冷(Zero Field 
Cooling, ZFC)的模式, 测量前先使样品零场降温至 $50 \mathrm{~K}$, 远低于铁磁/弱磁马氏体相变温度, 然后施加 $\mu_{0} H=0.1 \mathrm{~T}$ 的外磁场, 测量从 50 到 $400 \mathrm{~K}$ 的升温 $M(T)$ 曲 线, 这被称为 $\mathrm{ZFC}$ 过程; 然后 $\mu_{0} H=0.1 \mathrm{~T}$ 磁场下, 顺次测 量从 400 到 $50 \mathrm{~K}$ 的降温 $M(T)$ 曲线, 被称为 $\mathrm{FC}$ 过程. 等温 磁化 $M(H)$ 曲线采用循环(Loop)模式进行测量 ${ }^{[25]}$, 即测 量前先把样品零场降温至 $50 \mathrm{~K}$, 使之完全进入反铁磁 态, 然后升温到第一目标温度进行升降场 $M(H)$ 曲线的 测量，测试磁场范围为 $\mu_{0} H=0-5 \mathrm{~T}$, 然后再把样品降温 到 $50 \mathrm{~K}$, 再升温至下一个目标温度, 测量升降场 $M(H)$ 曲线, 然后重复此过程, 目的是获得准确的 $\Delta S_{\mathrm{M}}$ 值 ${ }^{[25]}$.

根据麦克斯韦关系式计算合金的 $\Delta S_{\mathrm{M}}$ 随温度和磁 场的变化关系 ${ }^{[14,15]}$ :

$$
\begin{aligned}
\Delta S_{\mathrm{M}} & =\mu_{0} \int_{0}^{\mu_{0} H}\left[\frac{\partial M\left(T, \mu_{0} H\right)}{\partial T}\right]_{\mu_{0} H} \mathrm{~d} H \\
& =\mu_{0} \frac{\partial}{\partial T} \int_{0}^{\mu_{0} H} M\left(T, \mu_{0} H\right) \mathrm{d} H .
\end{aligned}
$$

根据 $\Delta S_{\mathrm{M}}(T, H)$ 峰的半高宽所围的面积(数值积分) 计算合金的制冷能力(Refrigeration Capacity, $R C$ )值 ${ }^{[24]}$

$$
R C=\int_{T_{\text {cold }}}^{T_{\text {hot }}} \Delta S_{\mathrm{M}}\left(T, \mu_{0} \Delta H\right)_{\mu_{0} \Delta H} \mathrm{~d} T,
$$

其中, $T_{\mathrm{hot}}$ 和 $T_{\mathrm{cold}}$ 是 $\Delta S_{\mathrm{M}}(T, H)$ 峰的半高宽 $\left(\Delta T_{\mathrm{FWHM}}\right)$ 两端 点对应的温度值 ${ }^{[24]}$.

\section{3 结果和讨论}

图1为 $\mathrm{Cu} 1, \mathrm{Cu} 2$ 合金快淬条带和 $\mathrm{Cu} 1$ 退火条带的室 温XRD图谱. 如图所示, $\mathrm{Cu} 1$ 快淬条带主要为 5 层调制 结构 $(5 \mathrm{M})$ 的马氏体相与B2立方奥氏体相共存，且奥氏 体相为主相，这意味着合金的马氏体相变温度应该在 室温附近. 随着 $\mathrm{Cu}$ 取代量的增加， $\mathrm{Cu} 2$ 快淬条带的 $5 \mathrm{M}$ 马氏体相消失, 合金显示几乎单一的B2立方奥氏体相, 且B2(400)衍射峰强度异常增大, 预示着B2奥氏体相被 稳定，证明合金的马氏体相变随 $\mathrm{Cu}$ 取代量增加往低温 方向移动. 再看退火的影响，注意到Cu1退火条带，除 仍保持 $5 \mathrm{M}$ 马氏体和B2奥氏体相共存外, 出现非调制结 构的 $L 1_{0}$ 马氏体相, 这与前人的研究结果相符 ${ }^{[15,19,23]}$. 尽管三相共存, 但同快淬条带相比, 如图 1 右边 $2 \theta=63^{\circ}$ 附近局部放大的XRD图谱所示, B2(400)特征峰强度也 是异常增大, 表明退火后B2相被稳定, 比例增多, 预示

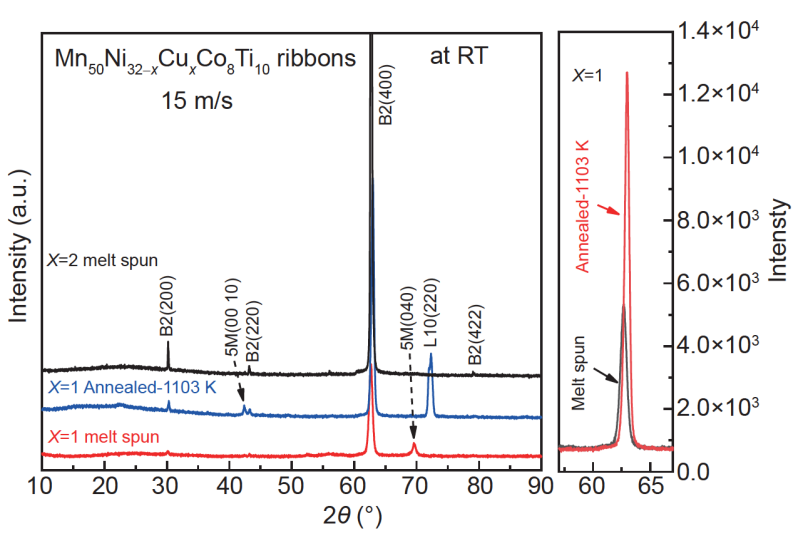

图 1 (网络版彩图) $\mathrm{Mn}_{50} \mathrm{Ni}_{32-x} \mathrm{Cu}_{x} \mathrm{Co}_{8} \mathrm{Ti}_{10}(x=1,2)$ 合金条带 的室温XRD图谱, 右边是 $2 \theta=63^{\circ}$ 附近B2(400)特征峰的局部 放大图

Figure 1 (Color online) XRD patterns for $\mathrm{Mn}_{50} \mathrm{Ni}_{32-x} \mathrm{Cu}_{x} \mathrm{Co}_{8} \mathrm{Ti}_{10}(x=1$, 2 ) alloy ribbons recorded at room temperature. The enlarged view of XRD pattern around $2 \theta=63^{\circ}$ is exhibited for $\mathrm{B} 2(400)$ characteristic peak.

着退火也会导致合金马氏体相变温度降低 ${ }^{[23]}$, 这与富 $\mathrm{Ni}$ 基all- $d$ 合金条带的情况完全相反 ${ }^{[18,19]}$ ，我们前期研 究表明, 退火会导致Co掺杂的 $\mathrm{Ni}_{50-x} \mathrm{Co}_{x} \mathrm{Mn}_{35} \mathrm{Ti}_{15}$ 合金体 系马氏体相被稳定，相变温度升高 ${ }^{[18,19]}$. 同时注意到, 富Mn基合金条带样品的B2(400)特征峰的强度非常强, 且在 $\mathrm{Cu}$ 取代和退火处理后, 峰强变得更是异常强, 这 在富 $\mathrm{Ni}$ 基all- $d$ 合金体系以及其他 Ni-Mn基Heusler合金 体系中都是很少报道的 ${ }^{[11,13,18,19]}$. 这些结果说明, 对富 $\mathrm{Mn}$ 基all- $d$ 合金条带，晶粒沿着(100)晶体学方向生长, 取向度非常高 ${ }^{[23]}$, 相关物理机制还需进一步研究.

图2为 $\mathrm{Cu} 1, \mathrm{Cu} 2$ 系列合金快淬条带在 $\mu_{0} H=0.1 \mathrm{~T}$ 磁 场下测量的 $M(T)$ 曲线, 不掺杂 $\mathrm{Cu}$ 的 $\mathrm{Mn}_{50} \mathrm{Ni}_{32} \mathrm{Co}_{8} \mathrm{Ti}_{10}$ (定 义为 $\mathrm{Cu} 0)$ 合金条带的 $M(T)$ 曲线也画出来以示比较 ${ }^{[23]}$, $\mathrm{Cu} 1$ 合金条带退火前后的 $M(T)$ 曲线放在插图中. 由图 可以看出, 所有的样品均表现出类似的马氏体相变行 为, 即随着温度的降低, 均发生从高温铁磁奥氏体到 低温弱磁马氏体的铁磁马氏体相变, 这与之前研究的 全过渡族金属基合金的相变行为相符很好 ${ }^{[14,15,18,19,23]}$, 证明 $\mathrm{Cu}$ 掺杂仍然可以实现剧烈的铁磁马氏体相变. 但 是相较于未掺杂 $\mathrm{Cu} 0$ 样品, $\mathrm{Cu}$ 掺杂后, 马氏体相变温度 往低温移动, 同时相变前后磁化强度突变量 $(\Delta M$, 定义 为逆马氏体相变开始温度 $A_{\mathrm{s}}$ 和结束温度 $A_{\mathrm{f}}$ 之间的磁化 强度差异)明显增大, 热滞 $\left(T_{\mathrm{hys}}\right.$ ) 先增大但马上几乎不再 变化. 相关的马氏体相变特征参数, 比如马氏体相变开 始温度 $\left(M_{\mathrm{s}}\right)$ 和结束温度 $\left(M_{\mathrm{f}}\right), A_{\mathrm{s}}, A_{\mathrm{f}}, T_{\mathrm{hys}}, \Delta M$ 等列在表 1 


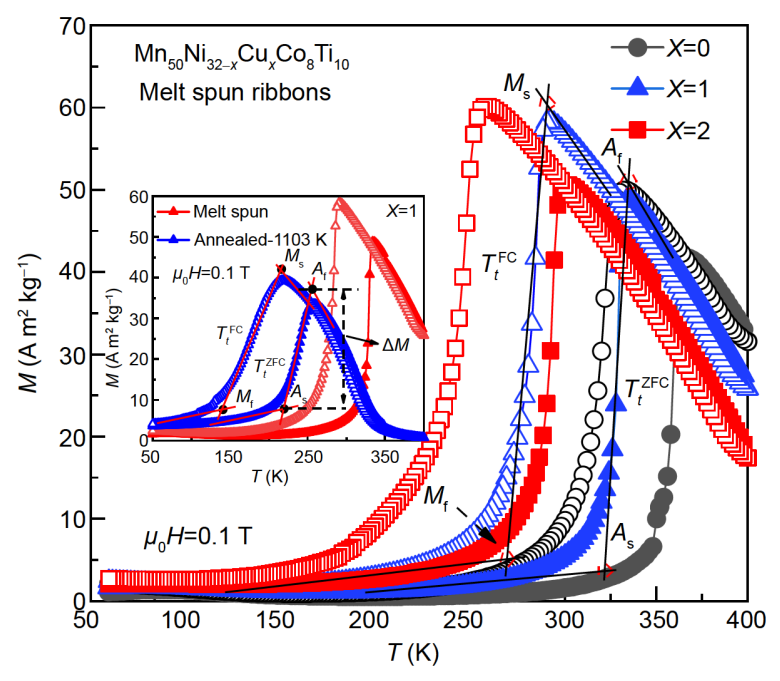

图 2 (网络版彩图) $\mathrm{Mn}_{50} \mathrm{Ni}_{32-x} \mathrm{Cu}_{x} \mathrm{Co}_{8} \mathrm{Ti}_{10}\left(x=0^{[23]}, 1,2\right)$ 合金 条带在 $\mu_{0} H=0.1 \mathrm{~T}$ 磁场下测量的热磁 $M(T)$ 曲线, 插图为 $x=1$ 时, 退火前后条带样品在 $\mu_{0} H=0.1 \mathrm{~T}$ 磁场下的 $M(T)$ 曲线

Figure 2 (Color online) Thermomagnetic $M(T)$ curves for $\mathrm{Mn}_{50^{-}}$ $\mathrm{Ni}_{32-x} \mathrm{Cu}_{x} \mathrm{Co}_{8} \mathrm{Ti}_{10}\left(x=0^{[23]}, 1,2\right)$ alloy ribbons under the applied field of $\mu_{0} H=0.1 \mathrm{~T}$. The inset shows the $M(T)$ curves of melt spun and annealed ribbons for $x=1$ sample under $\mu_{0} H=0.1 \mathrm{~T}$.

中. 马氏体相变和逆马氏体相变温度 $\left(T_{t}^{\mathrm{ZFC}}\right.$ 与 $\left.T_{t}^{\mathrm{FC}}\right)$ 定义 为ZFC和FC $M(T)$ 曲线一阶导数 $\mathrm{d} M / \mathrm{d} T(T)$ 的极大值对 应的温度. 由表1可知, 所有特征相变温度均明显降低. 这里面特别指出的是 $\mathrm{Cu}$ 取代部分 $\mathrm{Ni}$ 导致的相变温度 降低是非常敏感的, 比如逆马氏体相变温度 $T_{t}^{\mathrm{ZFC}}$, 从 $\mathrm{Cu} 0$ 到 $\mathrm{Cu} 1 ， 1$ at.\% $\mathrm{Cu}$ 的取代导致了 $28 \mathrm{~K}$ 温度的降低, 更有甚者, 从 $\mathrm{Cu} 1$ 到 $\mathrm{Cu} 2,1$ at.\% $\mathrm{Cu}$ 的取代竟然导致了 $36 \mathrm{~K}$ 的温度降低; 而马氏体相变温度 $T_{t}^{\mathrm{FC}}$, 从 $\mathrm{Cu} 0$ 到 $\mathrm{Cu} 2,1$ at.\% $\mathrm{Cu}$ 的取代均导致了 $38 \mathrm{~K}$ 的温度降低. 相较 于 $\mathrm{Cu}$ 取代, 退火对马氏体相变行为影响更为明显, 从 图2插图和表1可以看出，退火后，样品的马氏体相变 变得缓慢, $T_{\mathrm{hys}}$ 明显增大, $\Delta M$ 明显减小, $T_{t}^{\mathrm{ZFC}}$ 和 $T_{t}^{\mathrm{FC}}$ 分
别降低 84 和 $106 \mathrm{~K}$.

通过调研文献发现, $\mathrm{Cu}$ 对 $\mathrm{Ni}$ 的取代导致马氏体相 变往低温方向移动, 同 $\mathrm{Ni}_{42} \mathrm{Mn}_{36} \mathrm{In}_{14} \mathrm{Co}_{6} \mathrm{Cu}_{2}{ }^{[26]}$ 和 $\mathrm{Mn}_{50} \mathrm{Ni}_{40-x} \mathrm{Cu}_{x} \mathrm{In}_{10}{ }^{[27]}$ 相符. $\mathrm{Li}$ 等人 ${ }^{[27]}$ 认为 $\mathrm{Cu}$ 部分取代 $\mathrm{Ni}$ 导致 $3 d$ 巡游电子数目减少, $3 d$ 局域电子数目增加, 因此 $3 d$ 巡游电子和局域电子之间以及 $s$ 壳层和 $3 d$ 局域 电子之间的相互作用会受到影响. 具体到 $\mathrm{Mn}_{50} \mathrm{Ni}_{32} \mathrm{Co}_{8}$ $\mathrm{Ti}_{10}$ all- $d$ Heusler合金, Cu对Ni的取代可能还影响了 $\left(\mathrm{Mn}_{10} \mathrm{Ni}_{15-x} \mathrm{Co}_{x}\right)_{\mathrm{A}}\left(\mathrm{Mn}_{25}\right)_{\mathrm{B}}\left(\mathrm{Ni}_{25}\right)_{\mathrm{C}}\left(\mathrm{Ti}_{10} \mathrm{Mn}_{15}\right)_{\mathrm{D}}$ 原子相对占 位以及 $\mathrm{MnB}-\mathrm{CoA}-\mathrm{MnD}$ 原子构型 ${ }^{[15]}$, 肯定会影响过渡 元素之间的 $d-d$ 共价作用, 从而导致马氏体相变温度降 低和奥氏体铁磁作用增强. 当然, 进一步的研究还需通 过电子结构计算证明, 相关研究正在进行. 至于退火影 响引起马氏体相变行为的变化, 更多的原因还在于第二 相 $\gamma$ 相的析出 ${ }^{[11,13]}$, 为了进一步验证我们的想法, 本文观 察了合金条带的退火前后的微结构, 如图3所示.

本文以 $\mathrm{Cu} 1$ 为例, 分析退火引起马氏体相变行为 异常变化的原因. 如图3所示为在室温下 $\mathrm{Mn}_{50} \mathrm{Ni}_{31} \mathrm{Cu}_{1}$ $\mathrm{Co}_{8} \mathrm{Ti}_{10}$ 合金条带退火(在 $1103 \mathrm{~K}$ 时)前后的自由面和断 面的SEM微观结构图. 从图3(a)和(c)可以看出, 退火前 后, 条带样品均显示出等轴晶粒特征, 唯一的区别是, 退火样品等轴晶粒的晶界处有许多颗粒状析出物 $(\gamma$ 相)析出, 如图3(c)中红色虚线方框区域和插图(更大倍 数的SEM图)所示, 这与我们前面的研究相符 ${ }^{[23]}$. 利用 EDS对样品基体实际成分进行确定, $\mathrm{Cu} 1$ 快淬条带的平 均实际成分为 $\mathrm{Mn}_{48.0} \mathrm{Ni}_{30.8} \mathrm{Cu}_{1.8} \mathrm{Co}_{8.2} \mathrm{Ti}_{11.2}$, 退火后条带 的平均实际成分为 $\mathrm{Mn}_{54.8} \mathrm{Ni}_{26.1} \mathrm{Cu}_{1.6} \mathrm{Co}_{6.2} \mathrm{Ti}_{11.3}, \gamma$ 相的成 分通过点扫方法确定为 $\mathrm{Mn}_{56.9} \mathrm{Ni}_{24.9} \mathrm{Cu}_{1.6} \mathrm{Co}_{5.8} \mathrm{Ti}_{10.8}$. 通 过比较可以看出, $\gamma$ 相成分明显偏离基体成分. 另外, 退火条带成分相对于快淬条带也明显有所偏离，原因

表 $1 \mu_{0} H=0.1 \mathrm{~T}$ 外磁场下, $\mathrm{Cu} 1, \mathrm{Cu} 2$ 合金快淬条带与 $\mathrm{Cu} 1$ 退火条带的马氏体相变特征温度 $\left(M_{\mathrm{s}}, M_{\mathrm{f}}, A_{\mathrm{s}}, A_{\mathrm{f}}, T_{t}^{\mathrm{ZFC}}, T_{t}^{\mathrm{FC}}\right)$, 热滞 $\Delta T_{\mathrm{hys}}$ 以及逆马氏体相变前后磁化强度突变 $\Delta M$ 值

Table 1 The values of martensitic transformation characteristic temperatures $\left(M_{\mathrm{s}}, M_{\mathrm{f}}, A_{\mathrm{s}}, A_{\mathrm{f}}, T_{t}^{\mathrm{ZFC}}, T_{t}^{\mathrm{FC}}\right), \Delta T_{\text {hys }}$, and $\Delta M$ around the inverse martensitic transformation for $\mathrm{Cu} 1$ and $\mathrm{Cu} 2$ melt-spun and $\mathrm{Cu} 1$ annealed ribbons under $\mu_{0} H=0.1 \mathrm{~T}$

\begin{tabular}{ccccccccc}
\hline 条带样品 & $M_{\mathrm{s}}(\mathrm{K})$ & $M_{\mathrm{f}}(\mathrm{K})$ & $A_{\mathrm{s}}(\mathrm{K})$ & $A_{\mathrm{f}}(\mathrm{K})$ & $T_{t}^{\text {ZFC }}(\mathrm{K})$ & $T_{t}^{\mathrm{FC}}(\mathrm{K})$ & $\Delta T_{\mathrm{hys}}(\mathrm{K})$ & $\Delta M\left(\mathrm{~A} \mathrm{~m}{ }^{2} \mathrm{~kg}^{-1}\right)$ \\
\hline $\mathrm{Cu} 0$ 快淬 & 328 & 305 & 349 & 366 & 358 & 322 & 36 & 41.8 \\
$\mathrm{Cu} 1$ 快淬 & 288 & 266 & 322 & 332 & 330 & 284 & 46 & 47.2 \\
$\mathrm{Cu} 2$ 快淬 & 253 & 220 & 280 & 295 & 294 & 246 & 48 & 49.5 \\
$\begin{array}{c}\mathrm{Cu} 1 \text { 退火 } \\
(1103 \mathrm{~K})\end{array}$ & 215 & 137 & 216 & 254 & 246 & 178 & 68 & 30.2 \\
\hline
\end{tabular}

a) 数据来自于参考文献[23] 


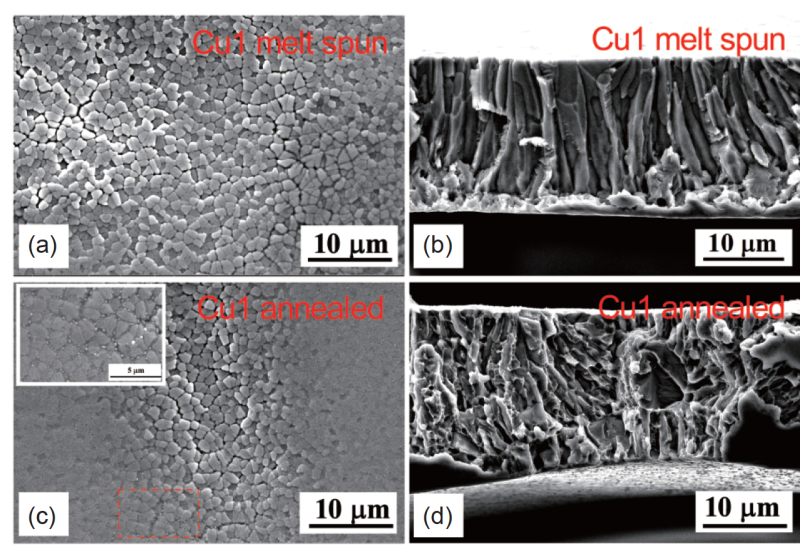

图 3 (网络版彩图)室温下观测的 $\mathrm{Mn}_{50} \mathrm{Ni}_{31} \mathrm{Cu}_{1} \mathrm{Co}_{8} \mathrm{Ti}_{10}$ 合金 快淬条带 $((a),(b))$ 和退火后条带( (c), (d))的表面((a), (c))和断 面((b), (d))SEM图. 插图表示图(c)中红色矩形区域的放大图 Figure 3 (Color online) Typical SEM micrographs observed at room temperature for the free surface ((a), (c)) and cross section ((b), (d)) of $\mathrm{Mn}_{50} \mathrm{Ni}_{31} \mathrm{Cu}_{1} \mathrm{Co}_{8} \mathrm{Ti}_{10}$ melt-spun ((a), (b)) and annealed ((c), (d)) ribbons. The inset denotes the enlarged view of red rectangle area in (c).

可能是EDS测量时选择的区域存在大量的析出相，导 致退火条带成分出现偏析. 由于退火和增大快淬速度 导致的 $\gamma$ 相析出分别在 $\mathrm{Ni}_{46} \mathrm{Mn}_{35} \mathrm{In}_{14} \mathrm{Co}_{5}{ }^{[11]}, \mathrm{Ni}-\mathrm{Co}-\mathrm{Mn}$ $\mathrm{Sn}$ 合金条带 ${ }^{[13]}$ 以及Ni-Fe-Mn-Sn微丝 ${ }^{[28]}$ 中也被观察到, 并且由此导致合金马氏体相变温度急剧降低和热滞增 大. 因此可以推断本文中 $\mathrm{Cu}$ 掺杂后的 $\mathrm{Mn}_{50} \mathrm{Ni}_{32-x} \mathrm{Cu}_{x}$ $\mathrm{Co}_{8} \mathrm{Ti}_{10}$ 合金马氏体相变温度降低和热滞增加的原因应 该也来自于 $\gamma$ 相的析出以及由此导致的合金主体成分 的偏析 ${ }^{[11,13,23,28]}$.

为了进一步研究合金马氏体相变行为, 本文对 $\mathrm{Cu} 1$ 和 $\mathrm{Cu} 2$ 快淬条带以及 $\mathrm{Cu} 1$ 退火条带在 $T_{t}^{\mathrm{ZFC}}$ 附近按照 升温次序测量了 $M(H)$ 曲线, 如图4所示, 磁场范围均为 $\mu_{0} H=0-5$ T. 从图 $4(\mathrm{a})$ 和(b)可以看出, $\mathrm{Cu} 1$ 和 $\mathrm{Cu} 2$ 快淬条 带表现出相似的等温磁化行为, 在远低于 $T_{t}^{\mathrm{ZFC}}$ 的温度 范围, 样品表现出弱磁性行为, 高于 $T_{t}^{\mathrm{ZFC}}$ 的温度区间 内, 样品表现出铁磁性行为, 在 $T_{t}^{\mathrm{ZFC}}\left(\mu_{0} H=5 \mathrm{~T}\right.$ 磁场下, $\mathrm{Cu} 1,322 \mathrm{~K} ; \mathrm{Cu} 2,288 \mathrm{~K}$ )附近, 样品实现了由磁场驱动 的剧烈变磁性马氏体相变, 磁化与退磁过程中明显的磁 滞证实了磁驱马氏体相变的一级性质, 预示着合金具有 大的磁热效应. 值得注意的是铁磁奥氏体饱和磁化强度 随 $\mathrm{Cu}$ 取代量的增加而增大, 比如 $\mu_{0} H=5 \mathrm{~T}$ 磁场下, $\mathrm{Cu} 1$ 在 $T=334 \mathrm{~K}$ 温度下的磁化强度值是62.7 $\mathrm{A} \mathrm{m}^{2} \mathrm{~kg}^{-1}$, 而 $\mathrm{Cu} 2$ 在 $T=296 \mathrm{~K}$ 温度下的磁化强度值是71.4 $\mathrm{A} \mathrm{m}^{2} \mathrm{~kg}^{-1}$, 这与 $M(T)$ 测量结果一致. 同时, 注意到随着 $\mathrm{Cu}$ 取代量增大, 合金快淬条带磁滞异常增大(图4). 通过调研文献发现, 这个现象应该与 $\mathrm{Cu}$ 取代量增大导致的奥氏体与马氏 体两相之间的几何相容性有关 ${ }^{[29]}$. 前人利用几何相容 性理论分析了马氏体相变中滞后变化的现象, 相变过 程中, 奥氏体与马氏体两相的几何相容性越好, 则滞 后越低, 反之亦然 ${ }^{[29]}$. 因此, 在本文的合金体系中, 当 $2 \%$ 的 $\mathrm{Cu}$ 取代 $\mathrm{Ni}$ 后, 有可能导致马氏体与奥氏体之间的 几何相容性减弱, 导致 $M(H)$ 曲线中显示的 $\mathrm{Cu} 2$ 快淬条 带在相变温度附近的磁滞明显大于 $\mathrm{Cu} 1$, 因而导致磁 滞损耗异常增大. 对于 $\mathrm{Cu} 1$ 退火后的样品, 虽然也观察 到具有一定的变磁性行为, 但要弱很多, 磁滞也减小很 多, 且铁磁奥氏体饱和磁化强度也相对退火前减小到 $53.1 \mathrm{~A} \mathrm{~m}^{2} \mathrm{~kg}^{-1}\left(\mu_{0} H=5 \mathrm{~T}, T=255 \mathrm{~K}\right)$, 这也与前述 $M(T)$ 测量结果一致.

基于上述 $M(H)$ 曲线，利用式(1)计算了合金的 $\Delta S_{\mathrm{M}}$ 值. 图5给出了 $\mathrm{Cu} 1$ 和 $\mathrm{Cu} 2$ 快淬条带以及 $\mathrm{Cu} 1$ 退火条带 在不同磁场变化下的 $\Delta S_{\mathrm{M}}(T)$ 图. 如图5(a)和(b)以及表2
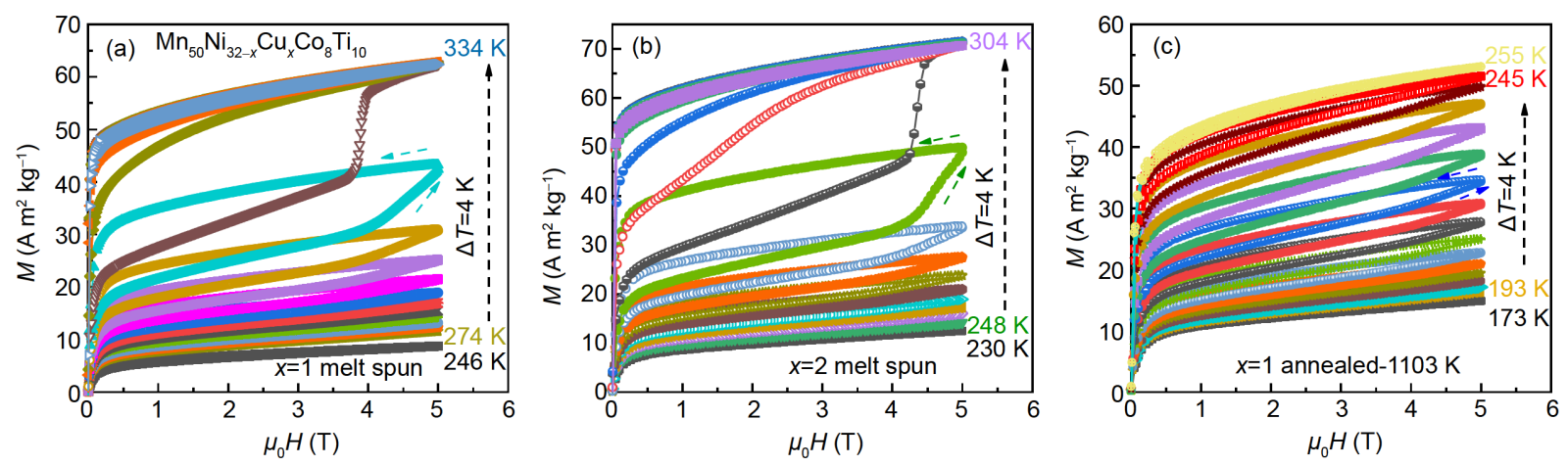

图 4 (网络版彩图) $\mathrm{Cu} 1$ (a), $\mathrm{Cu} 2$ (b)合金快淬与 $\mathrm{Cu} 1$ 退火条带(c)的等温磁化曲线. 箭头指示为磁化与退磁的过程

Figure 4 (Color online) Isothermal magnetization curves of $\mathrm{Cu} 1$ (a) and $\mathrm{Cu} 2$ (b) melt spun and $\mathrm{Cu} 1$ annealed ribbons (c). The arrows indicate the processes of magnetization and demagnetization. 

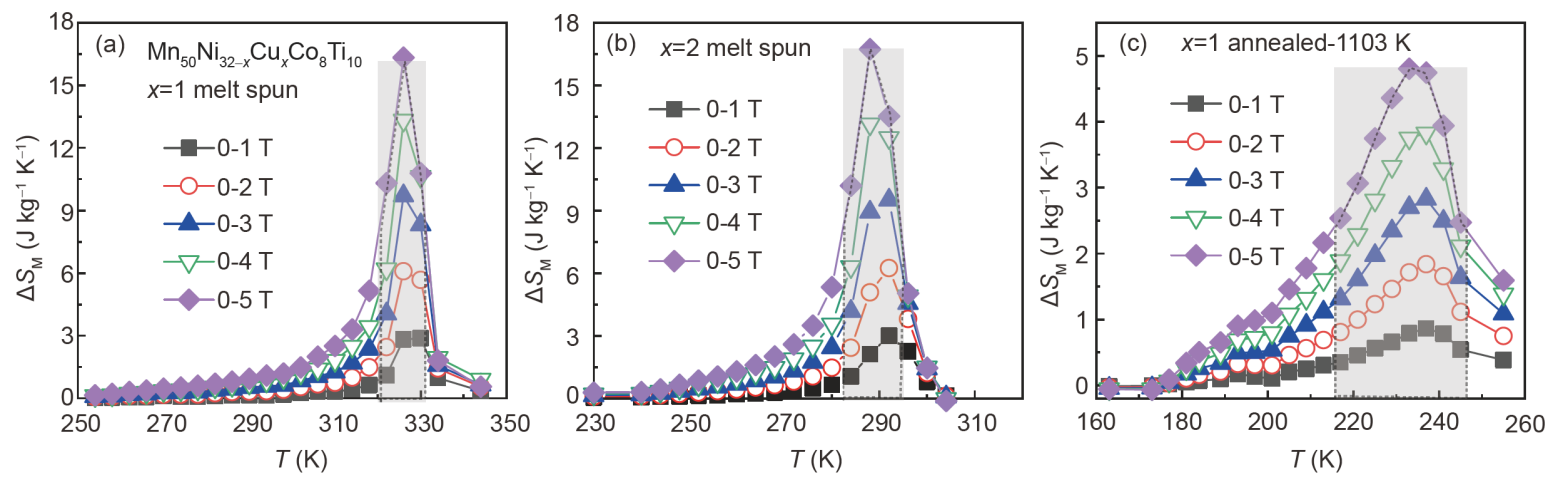

图 5 (网络版彩图)不同磁场变化下, $\mathrm{Cu} 1$ (a)和 $\mathrm{Cu} 2$ (b)合金快淬条带与 $\mathrm{Cu} 1$ 退火条带 $(\mathrm{c})$ 的 $\Delta S_{\mathrm{M}}$ 与温度的变化关系图

Figure 5 (Color online) Temperature dependence of $\Delta S_{\mathrm{M}}$ for $\mathrm{Cu} 1$ (a) and $\mathrm{Cu} 2$ (b) melt spun and $\mathrm{Cu} 1$ annealed ribbons (c) under different applied magnetic field changes.

所示, 相对 $\mathrm{Cu} 0$ 样品, 随着 $\mathrm{Cu}$ 取代量的增加，合金磁熵 变峰值 $\Delta S_{\mathrm{M}, \mathrm{MAX}}$ 增大, 但是对于 $\mathrm{Cu} 1$ 退火后条带, 明显可 以发现, $\Delta S_{\mathrm{M}, \mathrm{MAX}}$ 大幅度减小, 但是由于相变变缓(图2 插图), 导致半高宽温区几乎增大 3 倍. 为了正确评价合 金的磁热性能，我们还必须考虑磁场驱动的变磁性马 氏体相变产生的磁滞损耗 $(H L)^{[25]}$. 先利用式(2)计算出 合金的 $R C$ 值, 再通过 $M(H)$ 曲线中磁化与退磁过程所围 的闭合区域计算并画出 $H L$ 随温度变化曲线, 如图6所 示，然后根据计算 $R C$ 值的 $\Delta S_{\mathrm{M}}(T)$ 峰半高宽温度区间， 采用积分的方法计算出三个合金条带的平均磁滞损耗 $\left(H L_{\mathrm{ave}}\right)$ 值 ${ }^{[25]}$, 通过式子 $R C_{\mathrm{eff}}=R C-H L_{\mathrm{ave}}$ 扣除 $H L_{\mathrm{ave}}$, 得到 合金的 $R C_{\mathrm{eff}}$ 值. $R C, H L_{\mathrm{ave}}$ 和 $R C_{\mathrm{eff}}$ 值都列于表 2 中. 可以 看出, 相对 $\mathrm{Cu} 0, \mathrm{Cu}$ 掺杂后, $R C_{\mathrm{eff}}$ 值成倍增加, 即使对于 退火后条带, 尽管 $\Delta S_{\mathrm{M}, \mathrm{MAx}}$ 明显减小, 但由于相变变缓, 磁滞大幅度减小, 样品 $R C_{\text {eff }}$ 值退火前后基本保持不变.

\section{4 结论}



图 6 (网络版彩图) $\mathrm{Mn}_{50} \mathrm{Ni}_{32-x} \mathrm{Cu}_{x} \mathrm{Co}_{8} \mathrm{Ti}_{10}(x=1,2)$ 合金条带 磁滞损耗随温度变化曲线

Figure 6 (Color online) Temperature dependence of magnetic hysteresis loss for $\mathrm{Mn}_{50} \mathrm{Ni}_{32-x} \mathrm{Cu}_{x} \mathrm{Co}_{8} \mathrm{Ti}_{10}(x=1,2)$ alloy ribbons.

本文研究了全过渡族 $\mathrm{Mn}_{50} \mathrm{Ni}_{32-x} \mathrm{Cu}_{x} \mathrm{Co}_{8} \mathrm{Ti}_{10}(x=1,2)$ 合金条带 $\mathrm{Cu}$ 取代以及退火对马氏体相变和磁热效应

表 $2 \mu_{0} \Delta H=0-5$ T磁场变化下, $\mathrm{Cu} 1, \mathrm{Cu} 2$ 合金快淬条带与 $\mathrm{Cu} 1$ 退火条带的最大磁熵变 $\Delta S_{\mathrm{M}, \mathrm{MAX}}, \Delta S_{\mathrm{M}}(T)$ 峰的半高宽温度区间 $\Delta T_{\mathrm{FWHM}}$, 制冷能力 $R C$, 平均磁滞损耗 $H L_{\mathrm{ave}}$ 以及有效制冷能力 $R C_{\mathrm{eff}}$

Table 2 The magnetocaloric parameters: $\Delta S_{\mathrm{M}, \mathrm{MAX}}, \Delta T_{\mathrm{FWHM}}, R C, H L_{\text {ave }}, R C_{\text {eff }}$ for Cu1 and Cu2 melt-spun and Cu1 annealed ribbons under $\mu_{0} \Delta H=$ $0-5 \mathrm{~T}$

\begin{tabular}{|c|c|c|c|c|c|}
\hline 条带样品 & $\Delta S_{\mathrm{M}, \mathrm{MAX}}\left(\mathrm{J} \mathrm{kg}^{-1} \mathrm{~K}^{-1}\right)$ & $\Delta T_{\mathrm{FWHM}}(\mathrm{K})$ & $R C\left(\mathrm{~J} \mathrm{~kg}^{-1}\right)$ & $H L_{\text {ave }}\left(\mathrm{J} \mathrm{kg}^{-1}\right)$ & $R C_{\text {eff }}\left(\mathrm{J} \mathrm{kg}^{-1}\right)$ \\
\hline $\mathrm{Cu} 0$ 快淬 $\mathrm{a}^{\mathrm{a}}$ & 16.0 & 11 & 104.7 & 63.7 & 41.0 \\
\hline $\mathrm{Cu} 1$ 快淬 & 16.3 & 11 & 138.4 & 49.4 & 89.0 \\
\hline $\mathrm{Cu} 2$ 快淬 & 16.7 & 12 & 151.5 & 69.3 & 82.2 \\
\hline Cu1退火(1103 K) & 4.8 & 30 & 108.6 & 18.1 & 90.5 \\
\hline
\end{tabular}

a) 数据来自于参考文献[23] 
的影响. 首先无论 $\mathrm{Cu}$ 取代还是退火都导致马氏体相变 往低温方向移动, 且还都非常敏感, 但是 $\mathrm{Cu}$ 取代后, 马 氏体相变仍然保持很剧烈, 且 $\Delta M$ 逐渐增大; 而退火后, 合金的马氏体相变却变得很缓慢, $\Delta M$ 也减小. $\mathrm{Cu}$ 对 $\mathrm{Ni}$ 的取代可能影响了 $3 d$ 巡游电子和局域电子之间以及 $s$ 壳层和 $3 d$ 局域电子之间的相互作用，同时原子相对占
位和原子构型也会受到影响，进而影响了过渡元素之 间的 $d-d$ 共价作用, 从而导致马氏体相变温度降低和奥 氏体铁磁作用增强. 退火对马氏体相变行为的影响, 更 多的还在于 $\gamma$ 相析出导致的合金主体成分的偏析. 通过

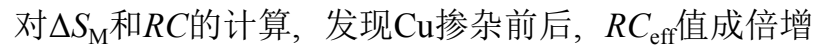
加, 而退火前后 $R C_{\text {eff }}$ 值基本保持不变.

\section{参考文献}

1 Liu F X, Zhang H, Zhou H, et al. Successive inverse and normal magnetocaloric effects in the Mn-vacancy compound $\mathrm{Mn}_{0.95} \mathrm{Co}_{0.75} \mathrm{Cu}_{0.25} \mathrm{Ge} \mathrm{Sci}$ China-Phys Mech Astron, 2020, 63: 277511

2 Xu L S, Wang R L, Yang C P, et al. Magnetic transition and magnetocaloric effect of $\mathrm{Mn}_{50} \mathrm{Ni}_{39} \mathrm{Sn}_{1-x} \mathrm{Al}_{x}$ alloys (in Chinese). Sci Sin-Phys Mech Astron, 2012, 42: 690-694 [徐莉莎, 王瑞龙, 杨昌平, 等. $\mathrm{Mn}_{50} \mathrm{Ni}_{39} \mathrm{Sn}_{1-x} \mathrm{Al}_{x}$ 合金的磁相变及磁卡效应. 中国科学: 物理学 力学 天文学, 2012, 42: 690-694]

3 Liu J, Gottschall T, Skokov K P, et al. Giant magnetocaloric effect driven by structural transitions. Nat Mater, 2012, 11: 620-626

4 Gottschall T, Gràcia-Condal A, Fries M, et al. A multicaloric cooling cycle that exploits thermal hysteresis. Nat Mater, 2018, 17: 929-934

5 Pecharsky V K, Gschneidner Jr. K A. Giant magnetocaloric effect in $\mathrm{Gd}_{5}\left(\mathrm{Si}_{2} \mathrm{Ge}_{2}\right)$. Phys Rev Lett, 1997, 78: 4494-4497

$6 \mathrm{Hu}$ F, Shen B, Sun J, et al. Influence of negative lattice expansion and metamagnetic transition on magnetic entropy change in the compound $\mathrm{LaFe}_{11.4} \mathrm{Si}_{1.6}$. Appl Phys Lett, 2001, 78: 3675-3677

7 Tegus O, Brück E, Buschow K H J, et al. Transition-metal-based magnetic refrigerants for room-temperature applications. Nature, 2002, 415: $150-152$

8 Li G J, Liu E K, Wang W H, et al. Structure, martensitic transformation and magnetism of $\mathrm{Ni}_{50-x} \mathrm{Mn}_{10+x} \mathrm{Ga}_{30} \mathrm{Cu}_{10}$ Heusler alloys (in Chinese). Sci Sin-Phys Mech Astron, 2012, 42: 677-683 [李贵江, 刘恩克, 王文洪, 等. $\mathrm{Ni}_{50-x} \mathrm{Mn}_{10+x} \mathrm{Ga}_{30} \mathrm{Cu}_{10}$ 系列Heusler合金的结构、马氏体相变和磁性 研究. 中国科学: 物理学 力学 天文学, 2012, 42: 677-683]

9 Sutou Y, Imano Y, Koeda N, et al. Magnetic and martensitic transformations of NiMnX(X=In,Sn,Sb) ferromagnetic shape memory alloys. Appl Phys Lett, 2004, 85: 4358-4360

10 Kainuma R, Imano Y, Ito W, et al. Magnetic-field-induced shape recovery by reverse phase transformation. Nature, 2006, 439: 957-960

11 Liu J, Woodcock T G, Scheerbaum N, et al. Influence of annealing on magnetic field-induced structural transformation and magnetocaloric effect in Ni-Mn-In-Co ribbons. Acta Mater, 2009, 57: 4911-4920

12 Mañosa L, González-Alonso D, Planes A, et al. Giant solid-state barocaloric effect in the Ni-Mn-In magnetic shape-memory alloy. Nat Mater, 2010, 9: 478-481

13 Ma S C, Shih C W, Liu J, et al. Wheel speed-dependent martensitic transformation and magnetocaloric effect in Ni-Co-Mn-Sn ferromagnetic shape memory alloy ribbons. Acta Mater, 2015, 90: 292-302

14 Wei Z Y, Liu E K, Chen J H, et al. Realization of multifunctional shape-memory ferromagnets in all-d-metal Heusler phases. Appl Phys Lett, 2015, 107: 022406, arXiv: 1507.05907

15 Wei Z Y, Liu E K, Li Y, et al. Magnetostructural martensitic transformations with large volume changes and magneto-strains in all-d-metal Heusler alloys. Appl Phys Lett, 2016, 109: 071904, arXiv: 1608.06301

16 Aznar A, Gràcia-Condal A, Planes A, et al. Giant barocaloric effect in all- $d$-metal Heusler shape memory alloys. Phys Rev Mater, 2019, 3: 044406

17 Cong D, Xiong W, Planes A, et al. Colossal elastocaloric effect in ferroelastic Ni-Mn-Ti alloys. Phys Rev Lett, 2019, 122: 255703

18 Liu K, Han X, Yu K, et al. Magnetic-field-induced metamagnetic reverse martensitic transformation and magnetocaloric effect in all-d-metal $\mathrm{Ni}_{36.0} \mathrm{Co}_{14.0} \mathrm{Mn}_{35.7} \mathrm{Ti}_{14.3}$ alloy ribbons. Intermetallics, 2019, 110: 106472

19 Liu K, Ma S, Ma C, et al. Martensitic transformation and giant magneto-functional properties in all- $d$-metal Ni-Co-Mn-Ti alloy ribbons. J Alloys Compd, 2019, 790: 78-92

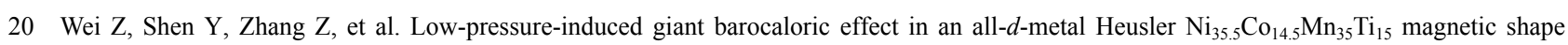


memory alloy. APL Mater, 2020, 8: 051101

21 Shen Y, Wei Z, Sun W, et al. Large elastocaloric effect in directionally solidified all- $d$-metal Heusler metamagnetic shape memory alloys. Acta Mater, 2020, 188: 677-685

22 Liu K, Ma S C, Zhang Z S, et al. Giant exchange bias effect in all-3 $d$-metal $\mathrm{Ni}_{38.8} \mathrm{Co}_{2.9} \mathrm{Mn}_{37.9} \mathrm{Ti}_{20.4}$ thin film. Appl Phys Lett, 2020, 116: 022412

23 Zhang Y X, Zeng H, Yu G, et al. Impact of annealing on the martensitic transformation and magnetocaloric properties in all-3d-metal $\mathrm{Mn}_{50} \mathrm{Ni}_{32} \mathrm{Co}_{8} \mathrm{Ti}_{10}$ alloy ribbons. Intermetallics, 2020, 125: 106882

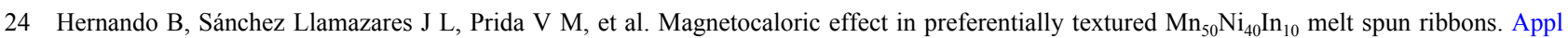
Phys Lett, 2009, 94: 222502

25 Caron L, Ba Doan N, Ranno L. On entropy change measurements around first order phase transitions in caloric materials. J Phys-Condens Matter, 2017, 29: 075401, arXiv: 1609.09831

26 Zhou Z N, Akamine H, Li J, et al. Tuning martensite transformation behavior and magneto-caloric effect in $\mathrm{Ni}_{44} \mathrm{Mn}_{36} \mathrm{In}_{14} \mathrm{Co}_{6}$ alloy through doping the fifth element $\mathrm{Cu}$. J Alloys Compd, 2020, 817: 153150

27 Li G T, Liu Z H, Meng F Y, et al. Effects of Cu on the martensitic transformation and magnetic properties of $\mathrm{Mn}_{50} \mathrm{Ni}_{40} \mathrm{In}_{10}$ alloy. Chin Phys B, 2013, 22: 126201

28 Zhang H, Qian M, Zhang X, et al. Magnetocaloric effect of Ni-Fe-Mn-Sn microwires prepared by melt-extraction technique. Mater Des, 2017, 114: 1-9

29 Cui J, Chu Y S, Famodu O O, et al. Combinatorial search of thermoelastic shape-memory alloys with extremely small hysteresis width. Nat Mater, 2006, 5: 286-290 


\title{
Martensitic transformation and magnetocaloric effect in $\mathrm{Mn}_{50} \mathrm{Ni}_{32-x} \mathrm{Cu}_{x} \mathrm{Co}_{8} \mathrm{Ti}_{10}$ Heusler alloy ribbons
}

\author{
ZHANG YuXi ${ }^{1}$, LIU XiaoChuan ${ }^{2}$, YU Guang ${ }^{1,2}$, ZHENG XianMing ${ }^{1,2}$, WAN DuoJian ${ }^{2}$, \\ LUO XiaoHua ${ }^{1}$, CHEN ChangCai ${ }^{1} \&$ MA ShengCan ${ }^{1 *}$ \\ ${ }^{1}$ Jiangxi Key Laboratory for Rare Earth Magnetic Materials and Devices/Institute for Rare Earth Magnetic Materials and Devices \\ (IREMMD), College of Rare Earths, Jiangxi University of Science and Technology, Ganzhou 341000, China; \\ ${ }^{2}$ Faculty of Materials Metallurgy and Chemistry, School of Materials Science and Engineering, Jiangxi University of Science and \\ Technology, Ganzhou 341000, China
}

The effects of $\mathrm{Cu}$ substitution and annealing on the martensitic transformation behavior and magnetocaloric properties in all- $d$-metal $\mathrm{Mn}_{50} \mathrm{Ni}_{32-x} \mathrm{Cu}_{x} \mathrm{Co}_{8} \mathrm{Ti}_{10}(x=1,2)$ alloy ribbons have been mainly investigated. After the substitution, it is observed that the martensitic transformation temperature of the alloys, which was extremely sensitive to the $\mathrm{Cu}$ content, was reduced. Moreover, the ferromagnetism of austenite increased, increasing the magnetization difference across the martensitic transformation. The magnetic-field-induced metamagnetic martensitic transformation also occurred. The magnetic entropy change gradually increased, and the effective refrigeration capacity increased by more than twice than that of the $\mathrm{Cu}$-free substitution. Moreover, the sample with $x=1$ was utilized as the research object to study the effect of annealing on the martensitic transformation. After annealing, the martensitic transformation became slow and the austenite ferromagnetism decreased. The change in the magnetization across the phase transition was also reduced. The magnetic-field-induced metamagnetic martensitic transformation was also weakened. Consequently, the magnetic entropy change was greatly reduced. However, the effective refrigeration capacity did not reduce significantly because the refrigeration temperature interval increased largely. In this paper, the physical mechanisms of the effects of $\mathrm{Cu}$ substitution and annealing on the martensitic transformation were discussed from the change in the $d$ - $d$ interaction and the precipitation in the $\gamma$-phase, respectively.

martensitic transformation, metamagnetic, magnetocaloric effect, $\gamma$ phase, $\mathrm{Mn}_{50} \mathrm{Ni}_{32-x} \mathrm{Cu}_{x} \mathrm{Co}_{8} \mathrm{Ti}_{10}$ alloy

PACS: $75.30 . \mathrm{Sg}, 75.20 . \mathrm{En}, 81.30 . \mathrm{Kf}, 75.60 . \mathrm{Nt}, 75.30 . \mathrm{Kz}$

doi: 10.1360/SSPMA-2020-0290 